\title{
Regular bulk solutions and black strings from dynamical braneworlds with variable tension
}

\author{
D. Bazeia* \\ Instituto de Física, Universidade de São Paulo, 05314-970 São Paulo, SP, Brazil \\ and Departamento de Física, Universidade Federal da Paraíba, 58051-970 João Pessoa, Paraíba, Brazil
}

J. M. Hoff da Silva ${ }^{\dagger}$

Departamento de Física e Química, Universidade Estadual Paulista, Avenida Doutor Ariberto Pereira da Cunha, 333 Guaratinguetá, São Paulo, Brazil

Roldão da Rocha

Centro de Matemática, Computação e Cognição, Universidade Federal do ABC, 09210-170 Santo André, SP, Brazil and International School for Advanced Studies (SISSA),

Via Bonomea 265, 34136 Trieste, Italy

(Received 21 March 2014; published 26 August 2014)

\begin{abstract}
Regular black strings solutions associated to a dynamical Friedmann-Robertson-Walker braneworld are obtained as a particular case of a bulk metric in the context of a variable tension braneworld. By analyzing the 5D Kretschmann invariants, we show that the variable brane tension is capable of attenuating the bulk physical singularities along some eras of the evolution of the Universe. nIn particular, the black string is analyzed for the McVittie metric on an Eötvös fluid braneworld, wherein the fluid dynamical brane tension depends on the brane temperature. The whole bulk is shown to be regular for a universe dominated by nonrelativistic matter or by relativistic matter/radiation, as the cosmological time elapses. In a particular case, the black strings are shown to have finite extension along the extra dimension, which prominently modify the higher-dimensional soft physical singularities. For a universe dominated by matter or radiation, we show that the bulk is regular as the cosmological time elapses, as an effect of the variable brane tension.
\end{abstract}

DOI: $10.1103 /$ PhysRevD.90.047902

PACS numbers: 11.25.-w, 04.50.-h, 04.50.Gh

\section{INTRODUCTION}

Braneworld models with variable brane tension are well-known probes for physical signatures arising from high energy physics. Indeed, the drastic modification of the temperature of the Universe along the cosmological evolution can be described by a braneworld model with variable tension, generalizing the original RandallSundrum model $[1,2]$, in order to allow Friedmann branes [3-5]. The variable tension brane dynamics was addressed in [6-9], where a brane tension with exponential dependence in the scale factor [10] was investigated. Moreover, black strings in Eötvös branes with variable tension were studied in $[11,12]$.

The field equations describing a brane containing any type of matter with a cosmological constant in the bulk were first studied in [4]. Some strategies for obtaining solutions with stable black holes on the brane have been extensively investigated [13]. Nevertheless, determining the bulk geometry is an extremely difficult task [14]. Computational methods designed to study braneworld relativistic stars and the exact solution of the collapse on

\footnotetext{
*bazeia@fisica.ufpb.br

†hoff@feg.unesp.br

†roldao.rocha@ufabc.edu.br
}

the brane [15], in the AdS/CFT correspondence framework $[16,17]$, are some tools to solve this question $[13,18]$.

Motivated by the relationship between braneworld black holes and the bulk metric [19,20], our main aim here is to study the black string and the bulk metric associated to a Schwarzschild black hole embedded in a FriedmannRobertson-Walker (FRW) variable tension braneworld. This can be accomplished by the McVittie metric [21]. McVittie solutions [22] lead to black hole metrics or the standard FRW cosmology in suitable limits, and are physically relevant for describing true black hole models [21,23-29]. We show, moreover, that the bulk can be regular in a variable brane tension model.

In this paper we analyze the McVittie black string warped horizon in a variable tension brane framework [11], in the three stages of the evolution of the Universe. We show that the black string may present finite extent along the extra dimension. The singularity structure of the higherdimensional spacetime has great importance in deciding whether a particular solution is physically acceptable. Therefore, we show by analyzing the 5D Kretschmann invariants that for a universe dominated by a cosmological constant, the 5D soft physical singularities on the brane remain in the bulk, where no further singularities are formed. Furthermore, the black string warped horizon vanishes in some point along the extra dimension when 
the Universe is dominated by matter or radiation. In this way the 5D physical singularities are alleviated due to the variable brane tension, leaving a regular 5D bulk.

\section{BULK METRIC AND BLACK STRING FROM A DYNAMICAL EÖTVÖS BRANEWORLD}

Let us consider a braneworld, with a single extra dimension of infinite extent, in a bulk endowed with a metric $\check{g}_{A B} d x^{A} d x^{B}=g_{\mu \nu}\left(x^{\alpha}, y\right) d x^{\mu} d x^{\nu}+d y^{2}$. The brane metric components $g_{\mu \nu}$ and the bulk metric are related by $\check{g}_{\mu \nu}=g_{\mu \nu}+n_{\mu} n_{\nu}$, where the $n^{\sigma}$ are timelike vector field components. Moreover, $\kappa_{4}^{2}=\frac{1}{6} \lambda \kappa_{5}^{4}$ and $\Lambda_{4}=$ $\frac{\kappa_{5}^{2}}{2}\left(\Lambda_{5}+\frac{1}{6} \kappa_{5}^{2} \lambda^{2}\right)$, where $\Lambda_{4}$ is the effective brane cosmological constant, $\kappa_{4}\left[\kappa_{5}\right]$ denotes the $4 \mathrm{D}$ [5D] gravitational coupling, and $\lambda$ is the brane tension. The extrinsic curvature

$$
K_{\mu \nu}=-\frac{1}{2} \kappa_{5}^{2}\left(T_{\mu \nu}+\frac{1}{3}(\lambda-T) g_{\mu \nu}\right)
$$

is obtained by using the junction conditions. $T^{\mu \nu}$ is the energy-momentum tensor.

The field equations together with the 5D Einstein and Bianchi equations $[13,19,30,31]$ are used to compute the bulk metric near the brane, and in particular the black string warped horizon $[3,32]$. Such a procedure provides information about the bulk metric components given by Eq. (10) of Ref. [11], and in particular it yields the black string warped horizon $g_{\theta \theta}\left(x^{\alpha}, y\right)$. In a variable brane tension scenario, the terms of polynomial order $|y|$ and $y^{2} / 2$ ! in Eq. (10) of Ref. [11] present no additional terms that could arise from the derivatives of the variable tension.
Notwithstanding, starting from the order $|y|^{3} / 3$ !, those additional terms play a fundamental role in the bulk metric. The term that contributes to the derivatives of the variable tension $\lambda$ in the order $|y|^{3} / 3$ ! reads

$$
-\frac{2 \kappa_{5}^{2}}{3}\left(\left(\nabla^{\alpha} \nabla_{\alpha} \lambda\right) g_{\mu \nu}-\left(\nabla_{(\nu} \nabla_{\mu} \lambda\right)\right) .
$$

Additional terms of order $y^{4} / 4$ !, arising from the variable tension brane, are given by Eq. (12) in [11].

The McVittie solution in isotropic spherical coordinates defined by $r=\mathrm{r}\left(1+\frac{2 G M}{\mathrm{r}}\right)^{2}[21,22]$ reads

$g_{\mu \nu} d x^{\mu} d x^{\nu}=\left(\frac{\mu-1}{\mu+1}\right)^{2} d t^{2}+(1+\mu)^{4} a^{2}(t)\left(d \mathrm{r}^{2}+\mathrm{r}^{2} d \Omega^{2}\right)$,

where $a(t)$ is the cosmological scale factor and $\mu=\frac{M}{2 a(t) r}$. The metric (3) is an exact solution of the Einstein field equations when the scale factor solves the Friedmann equation $\rho(t)=3 \dot{a}^{2} / 8 \pi G a^{2}$ for the energy density. The McVittie solution is the unique solution describing a spherically symmetric mass distribution in a spatially flat asymptotically FRW cosmology [26].

In order to analyze the black string associated to the McVittie metric, we focus on the term $g_{\theta \theta}\left(x^{\alpha}, y\right)$, as it represents the bulk metric near the brane and, in particular, the black string warped horizon. Clearly, a time dependent brane tension modifies the black string. By substituting the McVittie metric (3) in Eq. (10) of Ref. [11], denoting by $H(t)$ the Hubble parameter and $\beta=\frac{1-\mu}{1+\mu}$, it yields

$$
\begin{aligned}
g_{\theta \theta}(\mathrm{r}, t, y)= & \mathrm{r}^{2}\left\{1-\kappa_{5}^{2}\left(3 \frac{\dot{a}^{2}}{a^{2}}+\frac{\lambda}{3}+\frac{\dot{H}}{\beta}+\frac{3 \dot{a}^{2}}{2 \beta^{6}}\right)|y|+\left[\frac { 3 \dot { a } ^ { 4 } } { 1 6 a ^ { 4 } } \left[\frac{(2 \beta-1)^{2}}{6 \beta^{4}}+\frac{9 \beta^{2}}{4}-\frac{3 \beta}{2}+\frac{145}{4(1+\mu)^{4} a^{2}}+\left(\frac{3 \dot{a}^{2}}{a^{2}}+\frac{2 \dot{H}}{\beta}\right)\right.\right.\right. \\
& \left.\left.\left.\times\left(\frac{4 \mu^{2}+\mu+3}{(\mu-1)(1+\mu)^{4}}\right)\right]+\frac{\kappa_{5}^{4}}{36}\left(\lambda+\frac{3 \dot{a}^{2}}{2 \beta^{2}}(1+\mu)^{4}+\frac{9 \dot{a}^{2}}{2}+\frac{3 \dot{H}^{2}}{\beta}\right)+\frac{3 \beta}{2(1+\mu)^{4} a^{2}}+\frac{2 \lambda}{3}-\frac{\Lambda_{5}}{6}(1+\mu)^{4} a^{4}\right] \frac{y^{2}}{2 !}+\cdots\right\} .
\end{aligned}
$$

This expression is written up here in terms $y^{2} / 2$ ! for conciseness, although the awkward expansion including the term $y^{4} / 4$ ! was considered in [11], and shall be adopted in Figs. 1-4 hereupon. When $a(t)=1$ the bulk metric component is led to the Schwarzschild black string horizon, when $\mathrm{r}$ corresponds to the coordinate singularity $[11,13,23,33,34]$.

A natural choice to implement a variable brane tension is the fluid Eötvös brane [35], already used as a successful fluid braneworld model [6-8]. We assume the brane tension as an intrinsic property of the brane, just as an effective model [6-10]. The brane tension is also supposed to be smooth, to have a lower limit. Besides, the brane tension fluctuations are evanescent, in the sense that they are suppressed exponentially.
The huge variation of the temperature of the Universe in expansion needs to be modeled by a variable tension in the braneworld cosmology framework. The phenomenological Eötvös law [35] is therefore used, essentially,

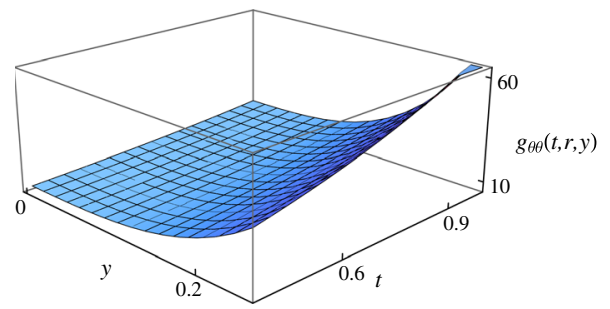

FIG. 1 (color online). Graphic of the black string warped horizon $g_{\theta \theta}(t, \mathrm{r}, y)$, with terms due to the variable brane tension, along $y$, as function of the time $t$. Here $a(t)=\exp \left(H_{0} t\right)$. 


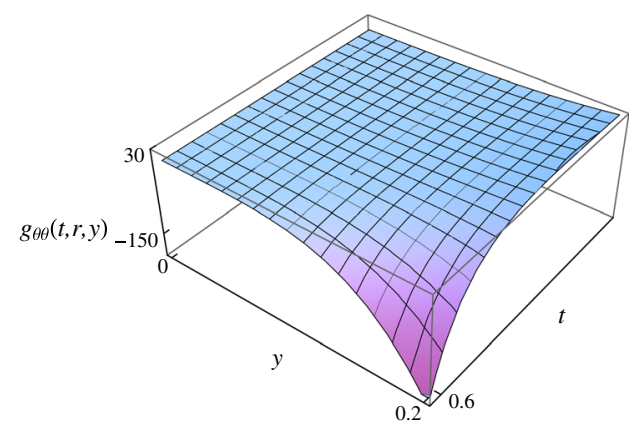

FIG. 2 (color online). Graphic of the black string warped horizon $g_{\theta \theta}(t, \mathrm{r}, y)$, with extra terms due to the variable brane tension along $y$, as function of the time $t$. Here $a(t) \propto t^{\beta / 2}$.

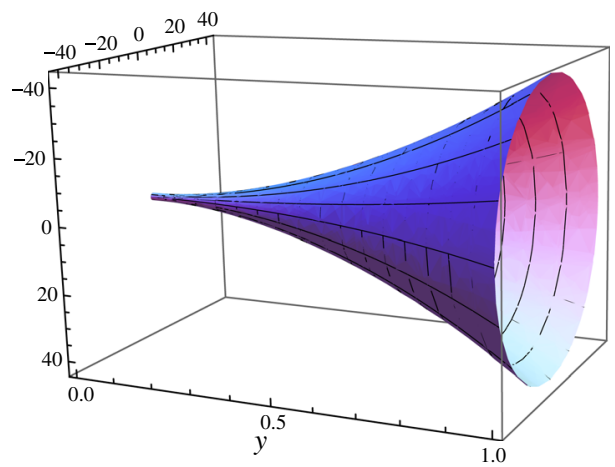

FIG. 3 (color online). Graphic of the black string for a universe dominated by relativistic matter or radiation, where $a(t) \propto \exp \left(H_{0} t\right)$.

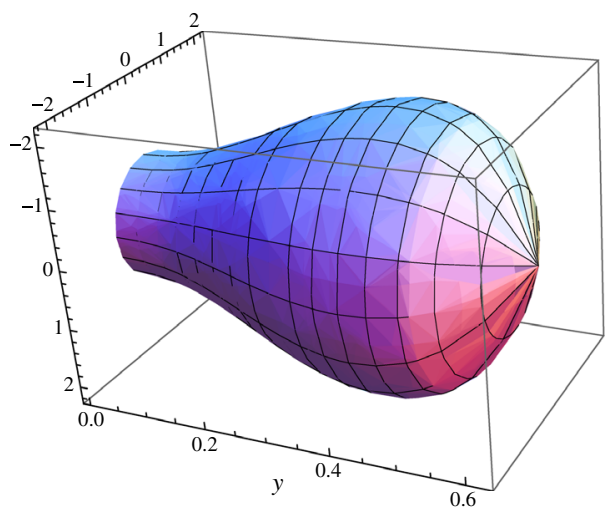

FIG. 4 (color online). Graphic of the black string for a universe dominated by relativistic matter or radiation, where $a(t) \propto t^{\beta / 2}$.

asserting that the fluid membrane tension depends on the temperature as

$$
\lambda=\chi\left(T_{c}-T\right),
$$

where $\chi$ is a constant and $T_{c}$ is a critical temperature above which the membrane does not exist. The tension variation is now expressed in terms of the (cosmic) time, instead of the temperature. Indeed, the Universe cools down as it expands, and a variation on the temperature corresponds to a time variation. In the absence of stresses in the bulk there is no exchange of energy-momentum between the brane and the bulk [13]. As $d Q=d E+p d V=0$, by taking into account photons from the cosmic microwave background, it is possible to use $E=\sigma T^{4} V$ and $p=E / 3$, implying that $\frac{d T}{T}=-\frac{1}{3} \frac{d V}{V}$. Finally, by expressing the volume $V(t) \propto a^{3}(t)$ it implies that $T(t) \propto 1 / a(t)$. This approach is in full agreement with the standard cosmological model [11]. Therefore Eq. (4) yields

$$
\lambda(t)=1-\frac{1}{a(t)},
$$

where both the brane tension and the scale factor are normalized.

By delving into the analysis on the influence of the brane tension variation on the McVittie black string, two points support our effective approach. Equation (5) is useful to merge supersymmetry and inflationary cosmology since it engenders a time variable 4D cosmological constant, which starts from a negative value and converges to a small positive one, as the Universe expands [7]. On the another hand, Eq. (5) is appropriate from the experimental point of view. The projection scheme of bulk gravitational quantities [30] implies a linear dependence between the effective Newtonian constant and the brane tension, namely, $G \sim \lambda$. Hence, a time variation on the brane tension means a time variable gravitational constant. The best model on the fractional variation of $G$ is provided by the Lunar Laser Ranging [36], asserting that $\dot{G} / G<(4.9 \pm 5.7) \times 10^{-13} y r^{-1}$. When the expression (5) is taken into account, the following bound is obtained:

$$
\frac{\dot{\lambda}(t)}{\lambda(t)}=\frac{\dot{a}(t)}{a(t)[a(t)-1]},
$$

and all the inputs here analyzed lead to a fractional variation of the brane tension.

Regarding the McVittie solution, the Einstein field equations on the brane provide $\rho \propto a^{-3(1+w) / \beta}$, where $w=$ $p / \rho$ is the state parameter. When $M=0$ the scale factor takes the well-known value for the scale factor of a flat universe $a(t) \propto t^{2 / 3}$ (dominated by nonrelativistic matter) or $a(t) \propto t^{1 / 2}$ (dominated by the radiation or relativistic matter). In the case of a cosmological constant, $a(t) \propto \exp \left(H_{0} t\right)$.

We now compare the McVittie black string profile in two eras of evolution of our Universe and, in addition, in the presence of a cosmological constant. The pure cosmological constant braneworld scenario is displayed in Fig. 1, approaching a realistic black string in a global asymptotically FRW braneworld. In this case, the scenario is dominated by a cosmological constant as the scale factor is given by $a(t) \propto \exp \left(H_{0} t\right)$. According to Eq. (5), the brane tension is given by $\lambda(t)=1-\exp \left(-H_{0} t\right)$. In the figures below we adopt $\Lambda_{5}=\kappa_{5}=1$. The case $a(t) \propto t^{\beta / 2}$, 
emulating a brane dominated by radiation, is also considered in Fig. 2, where Eq. (5) yields $\lambda(t)=1-t^{-\beta / 2}$.

There is a subtle and prominent difference between the warped horizons, regarding the respective corresponding eras. Figure 1 regards a brane dominated by a cosmological constant, and the horizon of the McVittie black string increases monotonically along the extra dimension. Notwithstanding, Fig. 2 shows that once the extra terms in Eq. (2) and Eq. (12) in [11] are taken into account, the effects are drastic. For $y \gtrsim y_{1} \sim 0.02$ the square of the black string horizon is negative, preventing the black string from existing for values greater than $y_{1}$.

The case of a matter-dominated FRW brane, where $a(t) \propto t^{2 \beta / 3}$, is very similar to the one in Fig. 2 . The only difference is that the warped horizon decreases along $y$ for any value of $t \lesssim 0.76$, and that there is a value $y=y_{1} \sim$ 0.018 above which the black string horizon ceases to exist. We illustrate below the McVittie black string, respectively for Fig. 1 and Fig. 2: The graphic for $a(t) \propto t^{2 \beta / 3}$ is completely similar to the case in Fig. 4. The variable brane tension scenario brings drastic changes in the black string profiles, as depicted in Fig. 3 and Fig. 4. In Fig. 3, the black string warped horizon always increases, in full compliance to Fig. 1. On the another hand, Fig. 4 shows that there is a point along the extra dimension where the black string horizon tends to zero, in accordance to Fig. 2. By analyzing the 5D Kretschmann invariants, the vanishing of the black string horizon shall be shown to correspond to a regular solution in the bulk.

\section{VARIABLE BRANE TENSION: REMOVING 5D PHYSICAL SINGULARITIES}

One of the natural ways to envisage the physical content of the bulk metric and of the black string is via the 4D and 5D Kretschmann invariants. Furthermore, we shall prove that for some eras of the evolution of the Universe, the bulk becomes regular as the cosmological time elapses, due to the variable brane tension.

Actually, in the case where at late times the cosmology is dominated by a positive cosmological constant, the metric (3) on the brane is regular everywhere on and outside the associated black hole horizon, and has asymptotically the Schwarzschild-de Sitter geometry [37]. When the cosmological constant equals zero, our results about the black hole horizons are led to the same results as the ones in [34]. When $M=0$, the solution is led to a homogeneous and isotropic FRW cosmology on the brane. For $H(t)=H_{0}$, the classical black string $[11,13,34]$ or a Schwarzschild-de Sitter black string [37] is obtained.

An alternative radial coordinate is defined [26] as $\mathfrak{r}=(1+\mu)^{2} a(t) \mathbf{r}$, and the McVittie metric (3) reads

$$
d s^{2}=-g d t^{2}-2 H \mathfrak{r} f^{-1 / 2} d \mathfrak{r} d t+f^{-1} d \mathfrak{r}^{2}+\mathfrak{r}^{2} d \Omega_{2},
$$

where $f=1-2 M / \mathfrak{r}$. On the brane, a null apparent horizon is placed at $\mathfrak{r}=\mathfrak{r}_{-}$, which is the smaller positive root of $g(\mathfrak{r})=1-2 M / \mathfrak{r}-H^{2} \mathfrak{r}^{2}=0$. When $\mathfrak{r}=2 M$ and $t$ is finite, the McVittie solution has a curvature singularity at $\mu=1$.

The invariant

$$
\xi=\left(\nabla_{\mu} \nabla_{\nu} R_{\phi \psi \rho \sigma}\right)\left(\nabla^{\mu} \nabla^{\nu} R^{\phi \psi \rho \sigma \sigma}\right)
$$

(here $\nabla_{\mu}$ denotes the covariant derivative on the brane) is very soft, since it involves at least two derivatives of the curvature. It can be related to its 5D counterpart, as the 5D and the 4D Riemann tensors are related by the Gauss equation as ${ }^{(5)} R_{\phi \kappa \rho \sigma}=R_{\phi \kappa \rho \sigma}-K_{\phi \rho} K_{\kappa \sigma}+K_{\phi \sigma} K_{\kappa \rho}$. Consequently, the 5D version of the invariant $\xi$ reads

$$
{ }^{(5)} \xi=\left(D_{a} D_{b}^{(5)} R_{\phi \kappa \zeta \sigma}\right)\left(D^{a} D^{b(5)} R^{\phi \kappa \zeta \sigma}\right),
$$

where $D_{a}$ denotes the 5D covariant derivative. Equation (7) diverges at the black string warped horizon as well as in the McVittie black string singularity [23], agreeing to the limit $a(t)=1$, corresponding to the classical black string.

The 4D Kretschmann scalar $\xi$ for the McVittie solution (3) encodes the existence of two physical soft singularities on the brane, at $\mathfrak{r}=0$ and $\mathfrak{r}=2 M$. The singularity at $\mathfrak{r}=2 M$ is a null naked singularity when $t \rightarrow \infty$ in a FRW brane if $H(t)$ goes to zero at latter times [21], which is the case when $a(t) \propto t^{\beta / 2}$ and $a(t) \propto t^{2 \beta / 3}$.

Equation (7) is a cumbersome expression for the McVittie solution, and we opt to depict it in the graphics in what follows. The figure for the scale factor $a(t) \propto t^{2 \beta / 3}$ is completely similar to Fig. 6. Moreover, Fig. 5 evinces that two physical singularities on the brane at $\mathfrak{r}=0$ and $\mathfrak{r}=2 M$ remain in the bulk and there is no additional singularity in the bulk, in a universe dominated by a cosmological constant. On the another hand, the vanishing of the black string warped horizon, for a universe dominated by matter or radiation (see Fig. 4) is accompanied by the attenuation of the soft physical singularities in the bulk. In fact, Fig. 6 shows that the 5D soft physical singularities in the bulk are alleviated as time elapses, providing a regular 5D bulk solution, as the 5D Kretschmann invariants do not diverge.

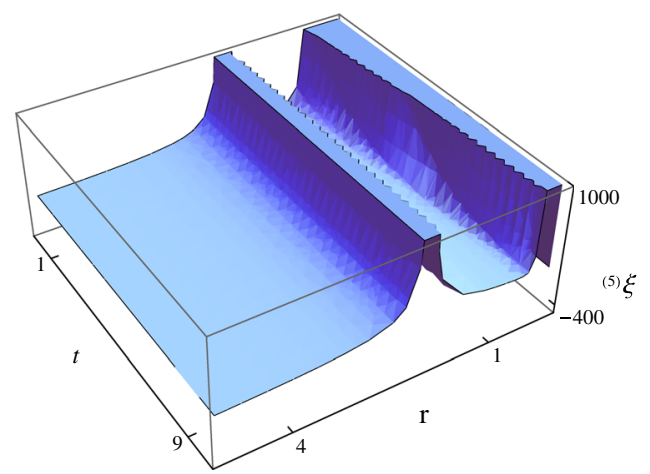

FIG. 5 (color online). Plot of the 5D Kretschmann scalar ${ }^{(5)} \xi$ as a function of time and $\mathfrak{r}$, for the scale factor $a(t) \propto \exp \left(H_{0} t\right)$. 


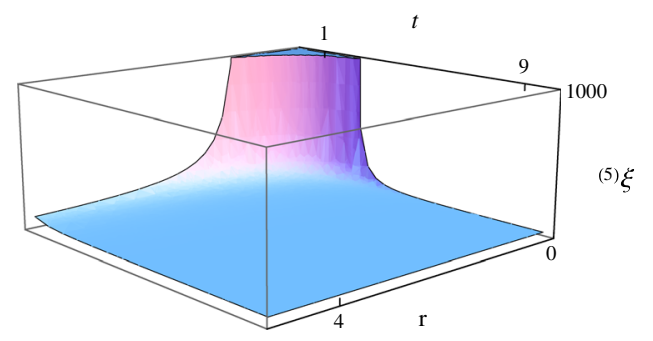

FIG. 6 (color online). Plot of the 5D Kretschmann scalar ${ }^{(5)} \xi$ as a function of time and $\mathfrak{r}$, for the scale factor $a(t) \propto t^{\beta / 2}$.

\section{CONCLUDING REMARKS}

The black string associated to the McVittie solution of the Einstein field equations is shown to be drastically modified by the terms due to the Universe expansion, in a variable tension braneworld scenario. When the variable brane tension is taken into account, the black string can cease to exist along the extra dimension for some range of time, as Fig. 4 illustrates. The black string warped horizon provides immediate information on the black string stability under small perturbations, as the black string Gregory-Laflamme instability [38]. Indeed, the horizon can collapse to zero before the perturbation takes part, as illustrated in Fig. 4.

By analyzing the 4D and 5D Kretschmann invariants, we have shown that the black string warped horizon vanishes along the extra dimension for a universe dominated by matter or radiation. It yields a regular bulk solution as the cosmological time elapses, due to the variable brane tension. The analysis of the bulk physical singularities relies on an exact method provided by the Gauss equation and by Eq. (7).

\section{ACKNOWLEDGMENTS}

The authors thank the referee of Phys. Rev. D for useful remarks and suggestions, and to Dr. R. Mosna for fruitful discussions. D. B. thanks CAPES, CNPq, and FAPESP for financial support. R. d. R. is grateful to CNPq Grants No. 303027/2012-6 and No. 473326/2013-2. R. d. R. is also Bolsista da CAPES Proc. $n^{\circ} 10942-13-0$. J. M. H. d. S. thanks CNPq.
[1] L. Randall and R. Sundrum, Phys. Rev. Lett. 83, 3370 (1999).

[2] L. Randall and R. Sundrum, Phys. Rev. Lett. 83, 4690 (1999).

[3] R. Maartens, Phys. Rev. D 62, 084023 (2000).

[4] P. Binetruy, C. Deffayet, U. Ellwanger, and D. Langlois, Phys. Lett. B 477, 285 (2000).

[5] D. Bazeia, F. A. Brito, and F. G. Costa, Phys. Lett. B 661 , 179 (2008).

[6] L. A. Gergely, Phys. Rev. D 78, 084006 (2008).

[7] L. A. Gergely, Phys. Rev. D 79, 086007 (2009).

[8] M. C. B. Abdalla, J. M. Hoff da Silva, and R. da Rocha, Phys. Rev. D 80, 046003 (2009).

[9] J. M. Hoff da Silva, Phys. Rev. D 83, 066001 (2011).

[10] K. C. Wong, K. S. Cheng, and T. Harko, Eur. Phys. J. C 68, 241 (2010).

[11] R. da Rocha and J. M. Hoff da Silva, Phys. Rev. D 85, 046009 (2012).

[12] R. Casadio, J. Ovalle, and R. da Rocha, Classical Quantum Gravity 31, 045016 (2014).

[13] R. Maartens and K. Koyama, Living Rev. Relativity 13, 5 (2010).

[14] C. G. Boehmer, G. De Risi, T. Harko, and F. S. N. Lobo, Classical Quantum Gravity 27, 185013 (2010).

[15] R. Casadio and J. Ovalle, Gen. Relativ. Gravit. 46, 1669 (2014).

[16] R. Emparan, A. Fabbri, and N. Kaloper, J. High Energy Phys. 08 (2002) 043.

[17] T. Tanaka, Prog. Theor. Phys. Suppl. 148, 307 (2002).

[18] H. Yoshino, J. High Energy Phys. 01 (2009) 068.

[19] L. A. Gergely, J. Cosmol. Astropart. Phys. 02 (2007) 027.
[20] E. Anderson and R. Tavakol, J. Cosmol. Astropart. Phys. 10 (2005) 017.

[21] N. Kaloper, M. Kleban, and D. Martin, Phys. Rev. D 81, 104044 (2010).

[22] G. C. McVittie, Mon. Not. R. Astron. Soc. 93, 325 (1933).

[23] D. Bazeia, J. M. Hoff da Silva, and R. da Rocha, Phys. Lett. B 721, 306 (2013).

[24] K. Lake and M. Abdelqader, Phys. Rev. D 84, 044045 (2011).

[25] V. Faraoni, A. F. Zambrano Moreno, and R. Nandra, Phys. Rev. D 85, 083526 (2012).

[26] B. C. Nolan, Phys. Rev. D 58, 064006 (1998).

[27] P. Haines and J. D. Harris, Astrophys. J. 418, 579 (1993).

[28] L. K. Patel, R. Tikekar, and N. Dadhich, Gravitation Cosmol. 6, 335 (2000).

[29] N. Sakai and P. Haines, Astrophys. J. 536, 515 (2000).

[30] J. Garriga and M. Sasaki, Phys. Rev. D 62, 043523 (2000).

[31] A. N. Aliev and A.E. Gumrukcuoglu, Classical Quantum Gravity 21, 5081 (2004).

[32] R. Casadio and C. Germani, Prog. Theor. Phys. 114, 23 (2005).

[33] S. S. Seahra, C. Clarkson, and R. Maartens, Phys. Rev. Lett. 94, 121302 (2005).

[34] A. Chamblin, S. W. Hawking, and H. S. Reall, Phys. Rev. D 61, 065007 (2000).

[35] R. Eötvös, Ann. Phys. (Berlin) 263, 448 (1886).

[36] C. M. Will, Living Rev. Relativity 9, 3 (2006).

[37] J. M. Hoff da Silva and R. da Rocha, Eur. Phys. J. C 72, 2258 (2012).

[38] R. Gregory and R. Laflamme, Phys. Rev. Lett. 70, 2837 (1993). 